\title{
Mortalidade perinatal em gestantes de alto risco em um hospital terciário
}

\section{Perinatal mortality in high-risk pregnant women in a tertiary hospital}

\author{
Rita de Cássia Alves Ferreira Silvaํ․ Pedro Sadi Monteiro². \\ 1. Serviço de Gestação de Alto Risco, Departamento de Obstetrícia, Hospital de Base Dr. Ary Pinheiro, Porto Velho, R0. 2. Programa de Pós-Graduação \\ em Ciências da Saúde, Faculdade de Ciências da Saúde, Universidade de Brasília, UNB, Brasília, DF.
}

\begin{abstract}
Resumo
Introdução: A mortalidade perinatal é um importante indicador de qualidade da atenção médica à gestante e ao recém-nascido. Este estudo teve como objetivo identificar possíveis causas de mortalidade perinatal em gestantes de alto risco em um hospital terciário de Porto Velho, Rondônia. Metodologia: Realizou-se um estudo transversal descritivo de julho a dezembro de 2010, com a amostra composta por 48 gestantes no período gestacional de 22 semanas ou mais. Os dados secundários foram coletados de prontuários e analisadas as seguintes variáveis: tipo e evolução do parto, indicadores obstétricos e evolução clínica dos recém-nascidos. Resultados: A taxa de mortalidade perinatal foi de 35,68/1000. A maioria dos partos ocorreu em ambiente hospitalar. Vinte e oito (60,9\%) partos ocorreram por via vaginal, com a faixa etária das gestantes variando entre 15 e 43 anos. Em relação à

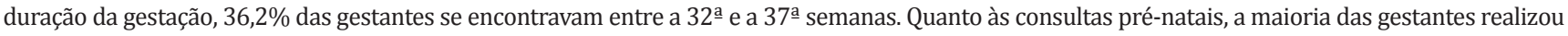
três ou quatro consultas, $42,3 \%$. Os diagnósticos maternos mais comuns foram infecção urinária (22,9\%) e trabalho de parto prematuro (22,9\%). Em relação ao diagnóstico dos produtos, 47,9\% eram natimortos e 20,8\% apresentaram malformação congênita. Conclusão: A taxa de mortalidade perinatal é elevada quando comparada com instituições similares. Infecção urinária e parto prematuro foram as causas mais frequentes de mortalidade perinatal, seguidas por eclâmpsia e coriamnionite.
\end{abstract}

Palavras-chave: Mortalidade perinatal. Causa Básica de Morte. Mortalidade Infantil. Gravidez de Alto Risco.

\begin{abstract}
Introduction: Perinatal mortality is an important indicator of quality of medical care to pregnant women and newborns. This study aimed at identifying possible causes of perinatal mortality in high-risk pregnancies in a tertiary hospital in Porto Velho, Rondônia. Methodology: A crosssectional study was performed from July to December 2010, with a sample of 48 pregnant women considering pregnancy period of 22 weeks or more. Secondary data were collected from medical records. The following variables were analyzed: type and evolution of childbirth, obstetric indicators and clinical evolution of newborns. Results: The perinatal mortality rate was 35.6/1000. The majority of the births occurred in hospitals. Twenty eight (60.9\%) of the births occurred vaginally, and the women`s age ranged from 15 to 43 years old. Regarding the extension of the pregnancy, 36.2\% of the women were between 32-37 weeks. As to prenatal visits, the majority of the women had had between three to four consultations, $42.3 \%$. The most common maternal diagnoses were urinary tract infections (22.9\%) and premature birth (22.9\%). Regarding diagnostic products, $47.9 \%$ were stillborn and $20.8 \%$ had malformation. Conclusion: The perinatal mortality rate is high when compared with similar institutions. Urinary tract infection and premature birth were the most frequent causes of perinatal mortality, followed by eclampsia and coriamnionite.
\end{abstract}

Descriptors: Perinatal mortality. Underlying Causes of Death. Infant Mortality. Pregnancy High-Risk.

\section{INTRODUÇÃO}

O conhecimento acerca do nível de mortalidade de uma população permite fazer inferências sobre as condições de saúde dos grupos que as constituem. O coeficiente de mortalidade perinatal é um indicador epidemiológico muito utilizado por obstetras e neonatologistas, pois se refere aos óbitos ocorridos a partir da 22a segunda semana de gestação até o 70 dia de nascimento do recém-nascido (RN). Ele inclui os natimortos e as crianças nascidas vivas, mas falecidas durante a primeira semana de vida ${ }^{1}$.
A mortalidade perinatal é reconhecida como o indicador mais apropriado para a análise da assistência obstétrica e neonatal. Recomenda-se a sua incorporação na rotina dos serviços de saúde de modo a dar visibilidade ao problema, propiciando a identificação das ações de prevenção, que podem alcançar ganhos mútuos na redução da morte fetal e neonatal precoce ${ }^{2}$.

O pronto reconhecimento do risco, com a provisão do cuidado apropriado e resolutivo à gestante (no pré-natal e

Conflito de interesses: Os autores declaram não ter conflito de interesses..

Correspondência: Rita de Cássia Alves Ferreira Silva. Serviço de Gestação de Alto Risco, Departamento de Obstetrícia, Hospital de Base Dr. Ary Pinheiro, Porto Velho, RO. e-mail: dracassiaferreira@hotmail.com

Recebido em 1 Dez 2013; Revisado em 25 Jan 2014; 4 Fev 2014. 
no parto) e à criança (na maternidade, na vigilância à saúde e por meio de assistência adequada à criança doente), são ações básicas com grande potencial para prover uma resposta mais positiva para a sobrevida e a qualidade de vida da criança ${ }^{3}$.

Os óbitos perinatais têm sido tratados como eventossentinela da qualidade da atenção médica e do sistema de saúde, pois sua ocorrência concentra-se cada vez mais na capacidade de atuação dos serviços de saúde. As principais causas da elevada mortalidade perinatal concentram-se na prematuridade, no baixo peso ao nascimento e nas infecções neonatais. Nos dois primeiros casos, os fatores demográficos, comportamentais e biomédicos têm a sua importância; no entanto, são os fatores socioeconômicos os mais contributivos e os que mais fogem do âmbito médico. A alta mortalidade, a elevada morbidade e as sequelas devem ser atribuídas às infecções neonatais. ${ }^{4,5}$.

Exposto isso, esse estudo tem como objetivo conhecer a magnitude da mortalidade perinatal em um hospital de referência em gravidez de alto risco no município de Porto Velho, Rondônia.

\section{METODOLOGIA}

Foi realizado um estudo do tipo transversal descritivo. A investigação da frequência dos óbitos perinatais abrangeu o período de julho a dezembro de 2010. O local da realização da pesquisa foi a Maternidade do Centro Obstétrico do Hospital de Base Dr. Ary Pinheiro, que é referência em gravidez de alto risco. É nessa maternidade que é prestada assistência materna e perinatal em nível terciário às gestantes do município de Porto Velho e das cidades vizinhas, incluindo a população ribeirinha da capital.

Foram incluídas todas as gestantes com óbitos perinatais e foram excluídas as gestantes com óbito fetal que tenham ocorrido após procedimento obstétrico invasivo (amniocetense, cordocentese e biópsia de vilo corial). Foram considerados natimortos os nascidos mortos com idade gestacional maior que 22 semanas e óbito neonatal precoce ou óbito infantil ocorrido com até sete dias completos de vida.

Foi realizada análise de prontuários dos óbitos perinatais ocorridos durante o período analisado, por meio do Serviço de Arquivos Médicos e Estatísticos do hospital. A análise dos dados foi realizada de forma descritiva, utilizando os programas Epi Info e Microsoft Excel.

Em relação ao parto, foram avaliadas as variáveis: local do parto, tipo de parto, presença de problemas durante o parto e tipo de nascimento; em relação à gestante, as variáveis são: idade, estado civil, idade gestacional, número de gestações anteriores, assistência pré-natal, comorbidades, renda financeira, escolaridade, profissão e raça; e em relação ao produto: gênero, peso e comorbidades.

Estudo aprovado pelo Comitê de Ética e Pesquisa da Faculdade de Ciências da Saúde da Universidade de Brasília, com registro do projeto $\mathrm{n}$ - 041/10.

\section{RESULTADOS}

No período de julho a dezembro de 2010, ocorreram 1.345 nascimentos, incluindo nascidos vivos e natimortos. Do total referido, registraram-se $48(3,6 \%)$ óbitos perinatais. Verificou-se que a taxa de mortalidade perinatal (TMP) foi de 35,6/1.000 nascidos.

Em relação às gestantes, verificou-se que a idade média foi de 22 anos, sendo a faixa etária mais comum entre 22 e 26 anos, 18 (37,5\%) gestantes. Em relação à duração da gestação, a idade gestacional variou entre 26 e 42 semanas, tendo $8(16,6 \%)$ gestantes acima de 37 semanas. Ao serem questionadas se realizaram consultas pré-natais, 45 (97,8\%) gestantes responderam que sim (Tabela 1 ).

Em relação às complicações clínicas durante a gravidez, 11 gestantes $(22,98 \%)$ apresentaram infecção urinária e $11(22,91 \%)$ tiveram trabalho de parto prematuro (Tabela 1). $35(85,4 \%)$ gestantes não eram fumantes, seis $(14,6 \%)$ gestantes eram fumantes, e sete foram ignoradas. Quanto à renda familiar, 18 (45\%) gestantes apresentaram renda entre 0 e 1 salário- mínimo, 7 (17,5\%) gestantes de 1 a 2 salários-mínimos, 6 (15\%) gestantes de 3 a 4 saláriosmínimos, 9 (22,5\%) gestantes acima de 5 salários mínimos e 10 foram ignoradas.

Quanto ao local do parto, 47 (97,9\%) gestantes realizaramno no centro obstétrico do hospital e uma $(2,1 \%)$ gestante teve o parto em sua residência. Em relação ao tipo do parto, $28(60,9 \%)$ gestantes foram submetidas ao parto por via vaginal e $18(39,1 \%)$ ao parto via cesárea. O transporte mais utilizado pela maioria das gestantes foram ambulâncias oriundas da capital ou das cidades do interior, $30(71,4 \%)$ gestantes (Tabela 2$)$. Quanto aos problemas durante o parto, $29(65,9 \%)$ gestantes não apresentaram intercorrências, enquanto 15 (34,1\%) gestantes apresentaram algum tipo de complicação, como apresentação pélvica, retenção placentária com curetagem pós-parto, hemorragia, mecônio espesso, estado pós-convulsional e infecção, e apenas quatro gestantes foram ignoradas. 
Tabela 1 Perfil social e diagnóstico das gestantes que evoluíram para óbitos perinatais.

\begin{tabular}{|c|c|c|}
\hline Variáveis & $\mathbf{n}$ & $\%$ \\
\hline \multicolumn{3}{|l|}{ Faixa etária } \\
\hline $15-21$ & 14 & 29,2 \\
\hline $22-26$ & 18 & 37,5 \\
\hline $27-35$ & 10 & 20,8 \\
\hline $35-43$ & 06 & 12,5 \\
\hline \multicolumn{3}{|l|}{ Duração da gestação } \\
\hline 22 a 25 semanas & 8 & 17,6 \\
\hline 26 a 31 semanas & 14 & 29,2 \\
\hline 32 e 36 semanas & 17 & 35,4 \\
\hline$\geq 37$ semanas & 08 & 16,7 \\
\hline \multicolumn{3}{|l|}{ Pré-natal } \\
\hline Sim & 45 & 97,8 \\
\hline Não & 1 & 2,2 \\
\hline \multicolumn{3}{|l|}{ Número de consultas pré-natais } \\
\hline 0 & 1 & 2,2 \\
\hline $1-2$ & 13 & 28,9 \\
\hline $3-4$ & 24 & 42,3 \\
\hline $5-6$ & 4 & 8,9 \\
\hline 7 ou mais & 3 & 2,2 \\
\hline \multicolumn{3}{|l|}{ Diagnóstico } \\
\hline Infecção urinária & 11 & 22,9 \\
\hline Trabalho de parto prematuro & 11 & 22,9 \\
\hline Pré-eclâmpsia & 10 & 20,8 \\
\hline Coriamnionite & 7 & 14,5 \\
\hline Incompetência istmo-cervical & 5 & 10,4 \\
\hline Hepatite viral & 1 & 2,1 \\
\hline
\end{tabular}

Tabela 2 Características relacionadas à ocorrência do parto de gestantes cujo produto evoluiu a óbito.

\begin{tabular}{ccc}
\hline Variáveis & $\mathbf{n}$ & $\%$ \\
\hline Local do parto & & 97,9 \\
Maternidade & 1 & 2,1 \\
Residência & & \\
Tipo de parto & 28 & 60,9 \\
Vaginal & 18 & 39,1 \\
Cesárea & & \\
Meio de transporte & & 71,4 \\
Ambulância & 30 & 14,3 \\
Carro & 6 & 9,5 \\
Ônibus & 4 & \\
\hline
\end{tabular}


Entre as características do produto, 24 (51,1\%) óbitos perinatais eram do gênero masculino. Como primeira causa de diagnóstico, obtiveram-se 23 (47,9\%) fetos como natimortos, $10(20,8 \%)$ fetos com alguma malformação congênita (onfalocele, hidrocefalia e malformação renal) e $6(12,5 \%)$ com septicemia. Quanto ao peso fetal, 14 (29,5\%) tinham peso menor que 2500 gramas (Tabela 3). tratamento a fim de evitar complicações no intraparto ${ }^{9}$.

O parto cesáreo não é isento de riscos. Ele pode contribuir para o aumento da morbimortalidade materna e fetal, além de acarretar elevação dos custos médico-hospitalares. Desse modo, a via de parto vaginal é classicamente a mais recomendada ${ }^{10,11}$. Neste estudo, 39,1\% das gestantes submeteram-se a partos do tipo cesariano.

Tabela 3 Características dos produtos que evoluíram para óbito perinatal.

\begin{tabular}{ccc}
\hline Variáveis & $\mathbf{n}$ & $\%$ \\
\hline Gênero & & 51,1 \\
Masculino & 24 & 48,9 \\
Feminino & 23 & 29,2 \\
Peso & & 70,8 \\
$\leq 2,5 \mathrm{Kg}$ & 14 & 52,1 \\
$\geq 2,5 \mathrm{Kg}$ & 34 & 47,9 \\
Óbitos Perinatais & & 47,9 \\
Óbito neonatal & 25 & 20,8 \\
Natimorto & 23 & 16,7 \\
Diagnóstico & & 12,5 \\
Natimorto & 23 & 2,1 \\
Malformação congênita & 10 & $\mathbf{1 0 0 , 0}$ \\
Prematuridade & 8 & 6 \\
Septicemia & 1 & \\
\hline Doença da membrana hialina & 48 & \\
\hline Total & & \\
\hline
\end{tabular}

\section{DISCUSSÃO}

O acompanhamento do sofrimento fetal durante o parto e o acesso à cesariana são medidas adotadas nos países desenvolvidos que contribuem para a redução da incidência de natimortos. ${ }^{6,7}$ No Brasil, a principal causa de óbitos em crianças abaixo de cinco anos são os problemas perinatais. Isso poderia ser evitado com melhorias na assistência perinatal durante a gestação, o intraparto e o período pós-parto8 . $^{\text {. }}$

Martins et al (2010) perinatal na cidade de Belo Horizonte, constatou que a TMP na cidade foi de 6,6/1000. Nesse estudo, verificou-se que a TMP foi de $35,68 / 1000$ nascidos, o que é bastante elevada quando comparada com esse estudo. A possível explicação para a taxa encontrada nesse estudo pode residir no fato de que, na instituição em que foi realizado o estudo, as gestantes de alto risco são assistidas; devido a essa condição, foi comum a detecção de gestantes que apresentaram complicações como hipertensão arterial sistêmica, bolsa rota, prematuridade, infecção urinária, diabetes e ausência e/ou número aquém de consultas no pré-natal. A elevada presença de doenças nas gestantes no período intraparto pode ser um reflexo de um pré-natal de baixa qualidade, em que essas doenças deveriam ter sido diagnosticadas e a gestante ter recebido seu devido
Laurenti e Buchala ${ }^{12}$, ao associar a idade materna ao evento da natimortalidade, constataram uma relação progressiva, ou seja, que o coeficiente de mortalidade era menor entre mães mais jovens, aumentando progressivamente com a idade e que, acima de 45 anos, o número de complicações era maior. Nesse estudo, foi evidenciado que a maioria das gestantes se encontrava na faixa etária entre 22 e 26 anos, não havendo nenhuma gestante com idade superior a 43 anos. Ao compararmos os óbitos perinatais pré-termo (abaixo de 37 semanas) e gestação a termo (acima de 37 semanas), observou-se que 39 gestantes apresentaram idade gestacional abaixo de 37 semanas, sendo a idade mínima de 22 semanas. A prevalência de prematuridade em revisão de estudos de base populacional variou de 3,4\% a 15,0\% nas regiões Sul e Sudeste, entre 1978 e 2004, sugerindo tendência crescente a partir da década de 1990, e na região Nordeste, entre 1994 e 1998, encontraram prevalências de prematuridade de 3,85 a 10,2\% ${ }^{13}$.

Ao analisar o número de consultas pré-natais, observou-se que a maioria, 24 (42,3\%) gestantes, realizou três ou quatro consultas, ficando, portanto, aquém do que é preconizado pela Organização Mundial da Saúde, seis ou mais consultas de pré-natal ${ }^{14}$. A insuficiência do número de consultas, bem como a precariedade da atenção pré-natal, têm-se mostrado 
fortemente associadas ao óbito neonatal, o que reforça a necessidade de um melhor atendimento à gestante ${ }^{14}$. A assistência pré-natal é um fator importante na redução da mortalidade perinatal, o que se compreende facilmente, visto que muitas patologias maternas que produzem efeitos sobre o feto podem ser tratadas ou controladas nesse período, evitando efeitos danosos para o produto.

Este estudo constatou que seis $(14,6 \%)$ das gestantes eram fumantes. Em um estudo realizado por Fonseca e Coutinho, em 2010, ao ser calculado o risco relativo da variável tabagismo e mortalidade fetal, verificou-se associação do óbito fetal com tabagismo em 134 pacientes estudadas ${ }^{15}$.

Nos países em desenvolvimento, a mortalidade infantil é elevada e uma significativa parcela desse número deve-se à mortalidade perinatal. As principais causas de mortalidade perinatal estão associadas à prematuridade, asfixia, infecções intrauterinas, toxemia gravídica e às malformações múltiplas e infecção pós-natal ${ }^{16-18}$. Andrade et al (2009) ${ }^{19}$, em seu estudo, evidenciaram maior risco de óbito fetal em pacientes que apresentaram síndromes hemorrágicas, trabalho de parto prematuro e malformações fetais. Nesse estudo, foi notado que as principais causas de mortalidade perinatal foram: infecção urinária e trabalho de parto prematuro. Atualmente, sabe-se que a utilização de práticas assistenciais pode reduzir a mortalidade perinatal, como o uso de corticoide no período antenal e o uso de surfactantes no pós-parto ${ }^{20}$. Nesse estudo, foi

\section{AGRADECIMENTOS}

Agradecemos a Gabriel de Deus Vieira do Departamento de Medicina, Faculdade São Lucas, Porto Velho, RO, pelas suas sugestões e auxílio na interpretação dos resultados.

\section{REFERÊNCIAS}

1. Camargo, ABM. A natimortalidade e a mortalidade perinatal em São Paulo. São Paulo perspect. [Internet]. 2008 Jan-Jun [acesso 2013 Nov 22];22(1):30-47. Disponível em: http://www.seade.gov.br/produtos/ spp/v22n01/v22n01_03.pdf.

2. Ministério da Saúde (BR). Manual dos Comitês de prevenção do óbito infantil e fetal [Internet]. Brasília: Ministério da Saúde; 2004 [acesso 2013 Nov 22]. 62 p. Disponível em:http://www.saude.rr.gov.br/sesau/ sistemas_visa/vigilancia_obito/manual_comites_maternoinfantil_gt/ manual_comite_poif_0001.pdf.

3. Silva ZP, Almeida MF, Ortiz LP, Alencar GP, Alencar AP, Shcoeps D et al. Morte neonatal precoce segundo complexidade hospitalar e rede SUS e não-SUS na Região Metropolitana de São Paulo, Brasil. Cad Saúde Pública [Internet]. 2010 Jan [acesso 2013 Nov 22];26(1):123134. Disponível em: Available from: http://www.scielo.br/scielo. php?script=sci_arttext\&pid=S0102-311X2010000100013\&Ing=en . doi: http://dx.doi.org/10.1590/S0102-311X2010000100013. verificado que $20,8 \%$ dos produtos apresentavam alguma malformação congênita. Esse tipo de complicação é, também, é uma importante de causa de morte perinatal, principalmente em países em desenvolvimento, sendo que sua evolução depende da qualidade e da disponibilidade da intervenção clínica e cirúrgica, além de uma prevenção primária, ou seja, o uso de ácido fólico antes ou durante os três primeiros meses de gestação, e um bom pré-natal ${ }^{8}$.

A presença de informações incompletas nos prontuário foi uma limitação deparada na execução desse estudo.

\section{CONCLUSÃO}

A TMP foi de 35,6/1000 nascidos, sendo o tipo vaginal o mais comum e o diagnóstico materno mais prevalente foi infecção urinária. As intervenções para reduzir a taxa mundial de natimortos, requerem ações em todos os níveis do sistema de saúde. Planos de intervenções devem ser adaptados às condições locais, incluindo, em nível local, as causas de morte fetal, a acessibilidade dos cuidados, os recursos do sistema de saúde e a habilidade do provedor. O pré-natal pode potencialmente servir como uma plataforma para realizar intervenções para melhorar a nutrição materna, promover a mudança de comportamento para reduzir a exposição nociva e o risco de infecções, além de tratar os fatores de risco da morbimortalidade maternoinfantil e incentivar a participação qualificada ao parto.
4. Bhutta ZA, Darmstadt GL, Haws RA, Yakoob MY, Lawn JE. Delivering interventions to reduce the global burden of stillbirths: improving service supply and community demand. BMC Pregnancy Childbirth [Internet]. 2009 May 7 [cited 2013 Nov 22];9 (Suppl 1):160187. Available from: http://www.ncbi.nlm.nih.gov/pmc/articles/ PMC2679413. doi: http://dx.doi.org/10.1186/1471-2393-9-S1-S7. Review. PubMed PMID: 19426470; PubMed Central PMCID: PMC2679413.

5. Martins EF, Lana FCF, Maria E. Tendência da Mortalidade perinatal em Belo Horizonte, 1984 a 2005. Rev Bras Enferm [Internet]. 2010 Jun [acesso 2013 Nov 22];63(3):446-451. Disponível em: http://www.scielo.br/scielo.php?script=sci_arttext \&pid=S003471672010000300016\&Ing=en. doi: http://dx.doi.org/10.1590/S003471672010000300016 .

6. Oliveira EFV, Gama SGN, Silva CFP. Gravidez na adolescência e outros fatores de risco para mortalidade fetal e infantil no Município do Rio de Janeiro, Brasil. Cad Saúde Pública [Internet]. 2010 Mar [acesso 2013 Nov 22];26(3):567-578. Disponível em: http://www.scielo.br/scielo. php?script=sci_arttext\&pid=S0102-311X2010000300014\&Ing=en. doi: http://dx.doi.org/10.1590/S0102-311X2010000300014.

7. Yao GM, Leone CR, Sadeck L, Vico ER, Taniguchi M. Sistema de investigação dos óbitos perinatais e neonatais por meio de comitês de mortalidade perinatal e infantil na cidade de São Paulo. Saúde Soc [Internet]. 2009 Mar [acesso 2013 Nov 22];18(Supl 1):80. Disponível em: http://www.scielo.br/scielo.php?script=sci_arttext\&pid=S010412902009000500032\&Ing=en. doi: http://dx.doi.org/10.1590/S010412902009000500032. 
8. Victora CG. Intervenções para reduzir a mortalidade infantil e pré-escolar e maternal no Brasil. Rev Bras Epidemiol [Internet]. 2001 Abr [acesso 2013 Nov 22];4(1):63-69. Disponível em: http://www.scielo.br/scielo. php?script=sci_arttext \&pid=S1415-790X2001000100002\&lng=en . doi: http://dx.doi.org/10.1590/S1415-790X2001000100002.

9. Rego MAS, França EB, Travassos APA, Barros FC. Avaliação do perfil de nascimentos e óbitos em hospital de referência. J Pediatr (Rio J) [Internet]. 2010 Jul-Ago [acesso 2013 Nov 22];86(4):295-302. Disponível em: http://www.jped.com.br/conteudo/10-86-04-295/ port.pdf. doi: http://dx.doi.org/10.1590/S0021-75572010000400009. PubMed PMID: 20711544.

10. Mandarino NR, Chein MBC, Monteiro Júnior FC, Brito LMO, Lamy ZC, Silva NVJ, et al. Aspectos relacionados à escolha do tipo de parto: um estudo comparativo entre uma maternidade pública e outra privada, em São Luís, Maranhão, Brasil. Cad Saúde Pública [Internet]. 2009 Jul [acesso 2013 Nov 22];25(7):1587-1596. Disponível em: http://www.scielo.br/scielo.php?script=sci_arttext\&pid=S0102311X2009000700017\&lng=en. doi: http://dx.doi.org/10.1590/S0102$311 \times 2009000700017$.

11. Ribeiro AM, Guimarães MJ, Carvalho M de L, Sarinho SW, Coutinho SB. Risk factors for neonatal mortality among children with low birth weight. Rev Saúde Pública [Internet]. 2009 Abr [acesso 2013 Nov 22];43(2):246-255. Disponível em: http://www.scielo.br/scielo. php?script=sci_arttext \&pid=S0034-89102009000200005\&Ing=en . Epub Feb 13, 2009. doi: http://dx.doi.org/10.1590/S003489102009005000004.

12. Laurenti R, Buchalla CM. Estudo da morbidade e da mortalidade perinatal em maternidades. I-Descrição do projeto e resultados gerais. Rev Saúde Pub [Internet]. 1984 Dez [acesso 2013 Nov 22];18(6):436447. Disponível em: http://www.scielo.br/scielo.php?script=sci_ arttext\&pid=S0034-89101984000600003\&lng=en. doi: http://dx.doi. org/10.1590/S0034-89101984000600003.

13. DATASUS (BR) MS/SVS/DASIS - Sistema de Informações sobre Mortalidade - SIM [base de dados na Internet]. Brasília: Ministério da Saúde. 2008 - Óbitos fetais / Dados preliminares / Notas técnicas; [acesso 2013 Nov 22]. Disponível em: http://tabnet.datasus.gov.br/ cgi/sim/pfet10descr.htm\#topo.

14. Silveira MF, Santos IS, Barros AJD, Matijasevich A, Barros FC, Victora CG. Aumento da prematuridade no Brasil: revisão de estudos de base populacional. Rev Saúde Pública [Internet]. 2008 Out [acesso 2013 Nov 22],42(5):957-964. Disponível em: http://www.scielo.br/scielo. php?script=sci_arttext\&pid=S0034-89102008000500023\&Ing=en . doi: http://dx.doi.org/10.1590/S0034-89102008000500023.
15. Fonseca SC, Coutinho ESF. Fatores de risco para Mortalidade fetal em uma Maternidade do Sistema Único de Saúde, Rio de Janeiro, Brasil: Estudo Caso-Controle. Cad Saúde Pública [Internet]. 2010 Fev [acesso 2013 Nov 22];26(2):240-252. Disponível em: http://www.scielo.br/scielo.php?script=sci_arttext\&pid=S0102311X2010000200004\&Ing=en. doi: http://dx.doi.org/10.1590/S0102$311 \times 2010000200004$.

16. Rasia ICRB, Albernaz E. Atenção pré-natal na cidade de Pelotas, Rio Grande do Sul, Brasil. Rev Bras Saúde Mater Infant [Internet]. 2008 Dez [acesso 2013 Nov 22];8(4):401-410. Disponível em: http://www.scielo.br/scielo.php?script=sci_arttext\&pid=S151938292008000400005\&Ing=en. doi: http://dx.doi.org/10.1590/S151938292008000400005 .

17. Carvalho PI, Pereira PMH, Frias PG, Vidal AS, Figueiroa JN. Fatores de risco para mortalidade neonatal em coorte hospitalar de nascidos vivos. Epidemiol Serv Saúde [Internet]. 2007 Set [acesso 2013 Nov 22],16(3):185-194. Disponível em: http://scielo.iec.pa.gov.br/pdf/ess/ v16n3/v16n3a05.pdf.

18. Fonseca SC, Coutinho ESF. Características biológicas e evitabilidade de óbitos perinatais em uma localidade na cidade do Rio de Janeiro, 1999 a 2003. Rev Bras Saúde Mater Infant [Internet]. 2008 Mar [acesso 2013 Nov 22];8(2):171-178. Disponível em: http://www.scielo.br/scielo. php?script=sci_arttext \&pid=S1519-38292008000200004\&Ing=en . doi: http://dx.doi.org/10.1590/S1519-38292008000200004.

19. Andrade LG, Amorim MMR, Cunha ASC, Leite SRF, Vital AS. Fatores associados à natimortalidade em uma maternidade escola em Pernambuco: estudo caso controle. Rev Bras Ginecol Obstet [Internet]. 2009 Jun [acesso 2013 Nov 22];31(6):285-292. Disponível em: http://www.scielo.br/scielo.php?script=sci_arttext\&pid=S010072032009000600004\&Ing=en. doi: http://dx.doi.org/10.1590/S010072032009000600004 .

20. Almeida MF, Guinsburg R, Martinez FE, Procianoy RS, Leone CR, Marba ST, et al. Perinatal factors associated with early deaths of preterm infants born in Brazilian Network on Neonatal Research centers. J Pediatr (Rio J) [Internet]. 2008 Jul-Aug [cited 2013 Nov 22];84(4):300-307. Available from: http://www.jped.com.br/conteudo/08-84-04-300/port.pdf. doi: doi:10.2223/JPED.1787. Epub 2008 May 26. PubMed PMID: 18528584.

Como citar este artigo / How to cite this article:

Silva RCAF, Monteiro PS. Mortalidade perinatal em gestantes de alto risco em um hospital terciário Perinatal mortality in high-risk pregnant women in a tertiary hospital. J Health Biol Sci. 2014 Jan-Mar; 2(1):22-27. 